\title{
Detection of Transport Intermediates in the Peptidoglycan Flippase MurJ Identifies Residues Essential for Conformational Cycling
}

Frederick A. Rubino, ${ }^{\prime \prime},+$ Aurelio Mollo, ${ }^{\|,+}$Sujeet Kumar, ${ }^{\perp}$ Emily K. Butler, ${ }^{\perp}$ Natividad Ruiz, ${ }^{*,}$ Suzanne Walker, ,* and Daniel E. Kahne, ${ }^{*}+$

${ }^{+}$Department of Chemistry and Chemical Biology, Harvard University, Cambridge, Massachusetts 02138, United States

${ }^{\ddagger}$ Department of Microbiology, Harvard Medical School, Boston, Massachusetts 02115, United States

${ }^{\perp}$ Department of Microbiology, Ohio State University, Columbus, Ohio 43210, United States

"These authors contributed equally to this work.

\section{SUPPLEMENTARY FIGURES AND TABLES}

Supplementary figure S1. Chemical structures of compounds used in this study 2

Supplementary figure S2. Chemical structures of native and biotinylated E. coli Lipid II 3

Supplementary figure S3. Uncropped western blots from Figure 1e $\quad 4$

Supplementary figure S4. Fosfomycin treatment depletes Lipid II levels $\quad \mathbf{5}$

Supplementary figure S5. Uncropped western blots from Figure 2a $\quad 6$

Supplementary figure S6. Uncropped western blots from Figure 2b $\quad 7$

Supplementary figure S7. Uncropped western blots from Figure 2c $\quad \mathbf{8}$

Supplementary figure S8. Uncropped western blots from Figure 2d 9

Supplementary figure S9. MurJ and MurJXLipid II can be resolved by SDS-PAGE 10

Supplementary figure S10. Uncropped western blots from Figure 3b 11

Supplementary figure S1 1. Treatment with $1 \mu \mathrm{M}$ CCCP does not accumulate MurJ×Lipid II 12

Supplementary figure S12. Uncropped western blots from Figure 3d 13

Supplementary figure S13. The diffuse membrane protein GlpT does not crosslink to Lipid II $\quad 14$

Supplementary figure S14. Uncropped western blots from Figure 4c 15

Supplementary figure S15. Uncropped western blots from Figure 4d 16

$\begin{array}{lr}\text { Supplementary table S1. Strains used in this study } & 17\end{array}$

$\begin{array}{lr}\text { Supplementary table S2. Plasmids used in this study } & 18\end{array}$

\section{SUPPLEMENTARY TEXT}

$\begin{array}{lr}\text { Materials and methods } & 19\end{array}$

$\begin{array}{lr}\text { References } & 22\end{array}$ 
Supplementary figure S1. Chemical structures of compounds used in this study.

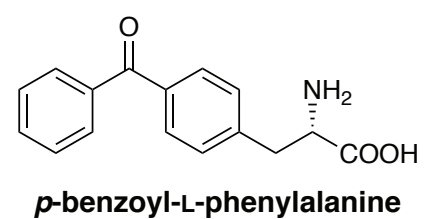

(pBpa)

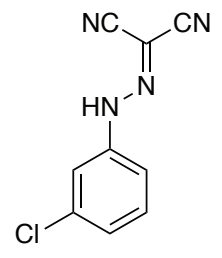

carbonyl cyanide $\boldsymbol{m}$-chlorophenyl hydrazone (CCCP)

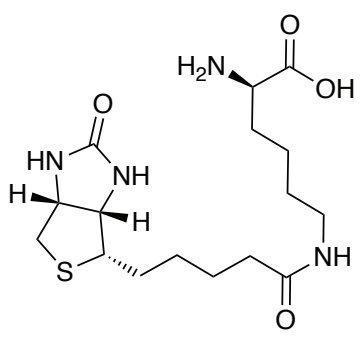

biotin-D-Lysine

(BDL)

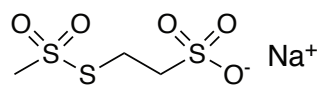

Sodium (2-sulfonatoethyl)methanethiosulfonate (MTSES)

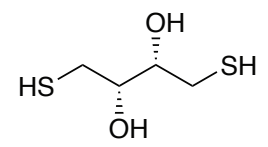

DL-dithiothreitol (DTT)

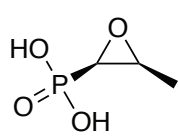

fosfomycin 
Supplementary figure S2. Chemical structures of native and biotinylated E. coli Lipid II.

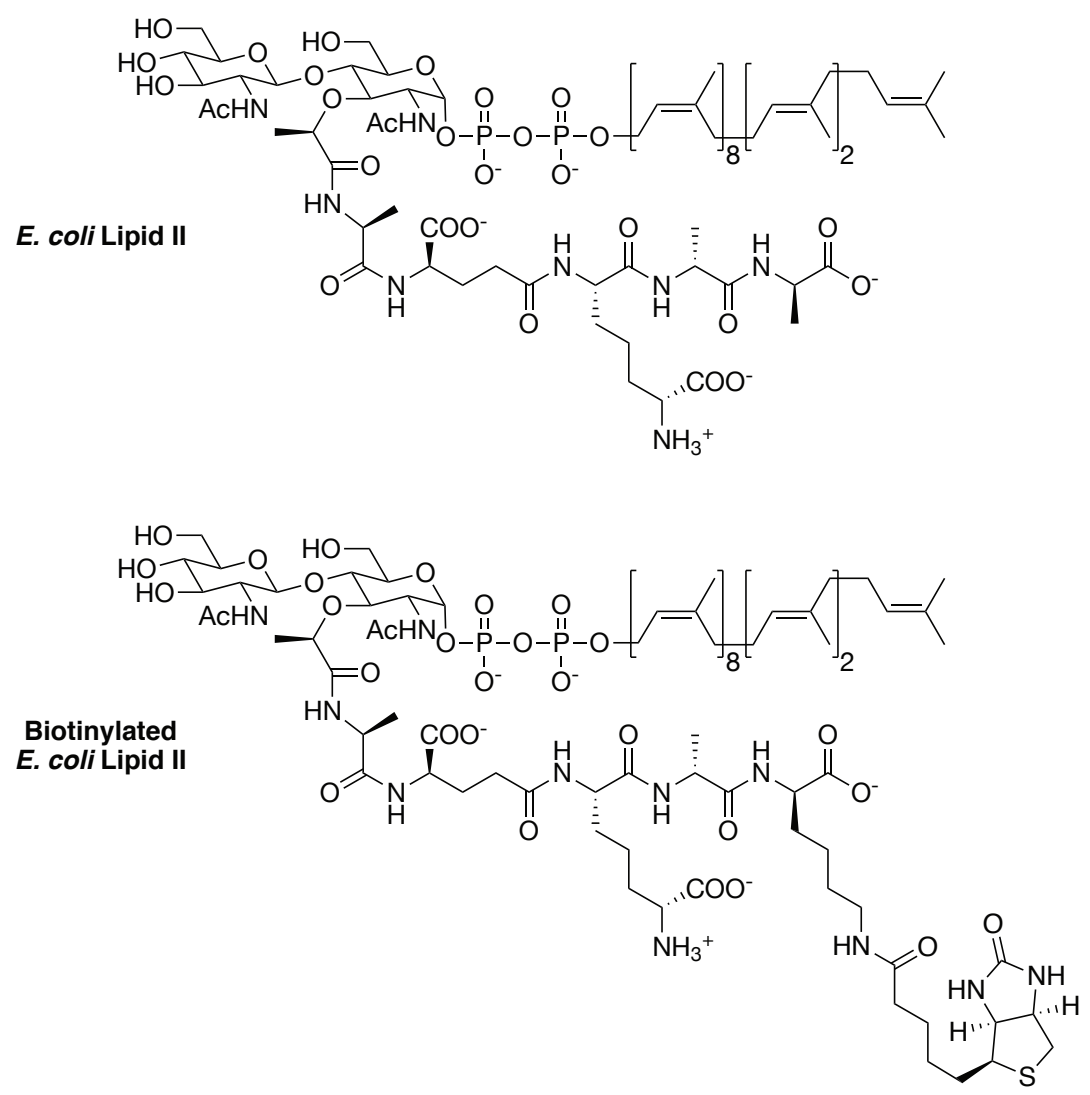


Supplementary figure S3. Uncropped western blots from Figure 1e.

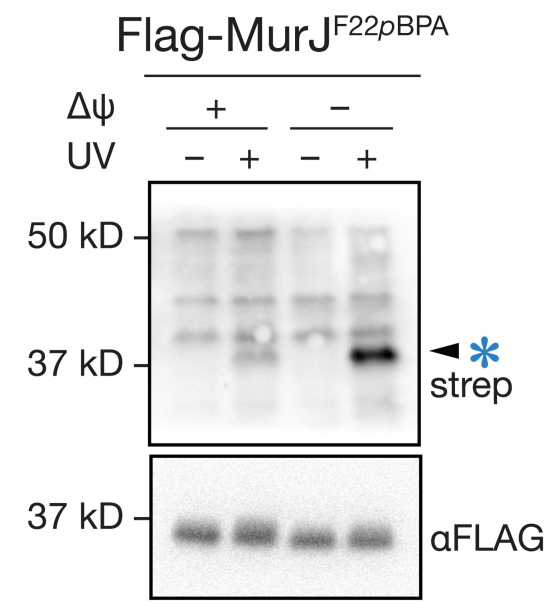


Supplementary figure S4. Fosfomycin treatment depletes Lipid II levels. A culture of NR5684 was grown to OD 0.3 in M63 minimal medium and resuspended to OD 1.0. Sucrose was added to $0.23 \mathrm{M}$ final concentration, followed by $100 \mu \mathrm{g} / \mathrm{mL}$ fosfomycin, and the cells incubated at $37^{\circ} \mathrm{C}, 550 \mathrm{rpm}$ on Thermomixer $\mathrm{C}^{\varpi} .100 \mu \mathrm{L}$ aliquots were taken after the specified time periods for Lipid II quantification.

\begin{tabular}{|c|c|c|c|c|c|c|c|c|c|}
\hline Fosfomycin & - & & & & + & & & & \\
\hline Treatment time (min) & & 5 & 10 & 20 & 30 & 45 & 60 & 90 & \\
\hline
\end{tabular}


Supplementary figure S5. Uncropped western blots from Figure 2a.

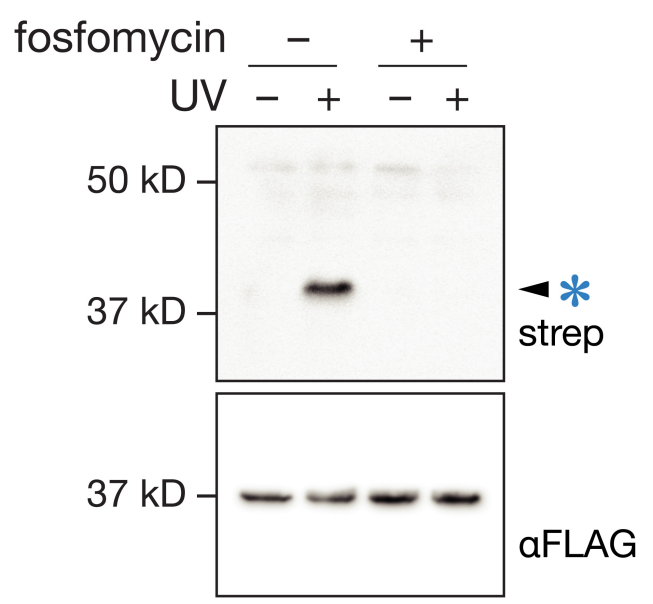


Supplementary figure S6. Uncropped western blots from Figure 2b.

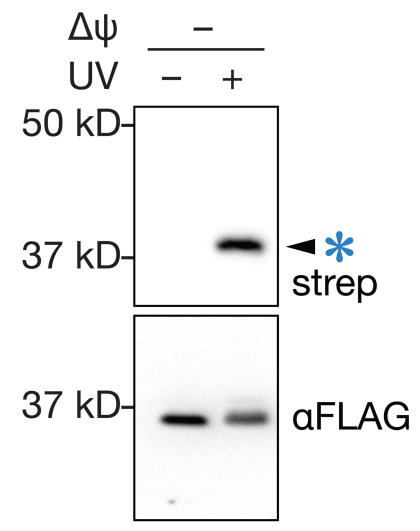


Supplementary figure S7. Uncropped western blots from Figure 2c.

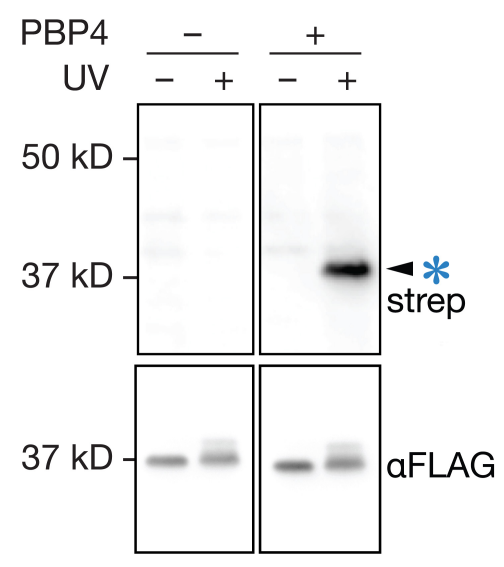


Supplementary figure S8. Uncropped western blots from Figure 2d.

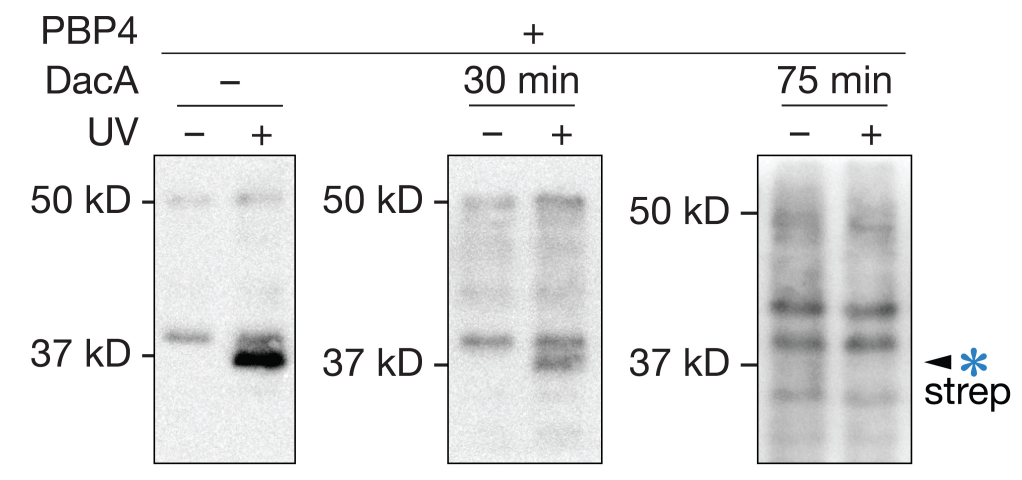


Supplementary figure S9. MurJ and MurJ×Lipid II can be resolved by SDS-PAGE. Crosslinked Flag-MurJ ${ }^{\mathrm{F} 22 p \mathrm{Bpa}}$ sample was run on a $10 \%$ gel (4\% stacking layer) and transferred to a PVDF membrane. Following onmembrane biotinylation, visualization was performed using a mixture of the IR-dye-tagged M2 ${ }^{\circledR}-700(2 \mu \mathrm{g} / \mathrm{mL})$ and Strep-800 $(1 \mu \mathrm{g} / \mathrm{mL})$.

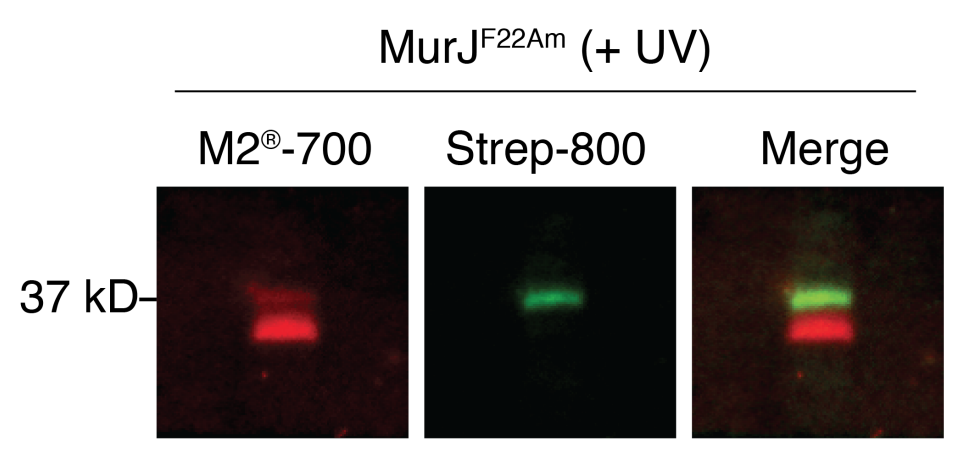


Supplementary figure S10. Uncropped western blots from Figure 3b, including biological replicates and quantification of MurJ $\times$ Lipid II content for the third replicate.

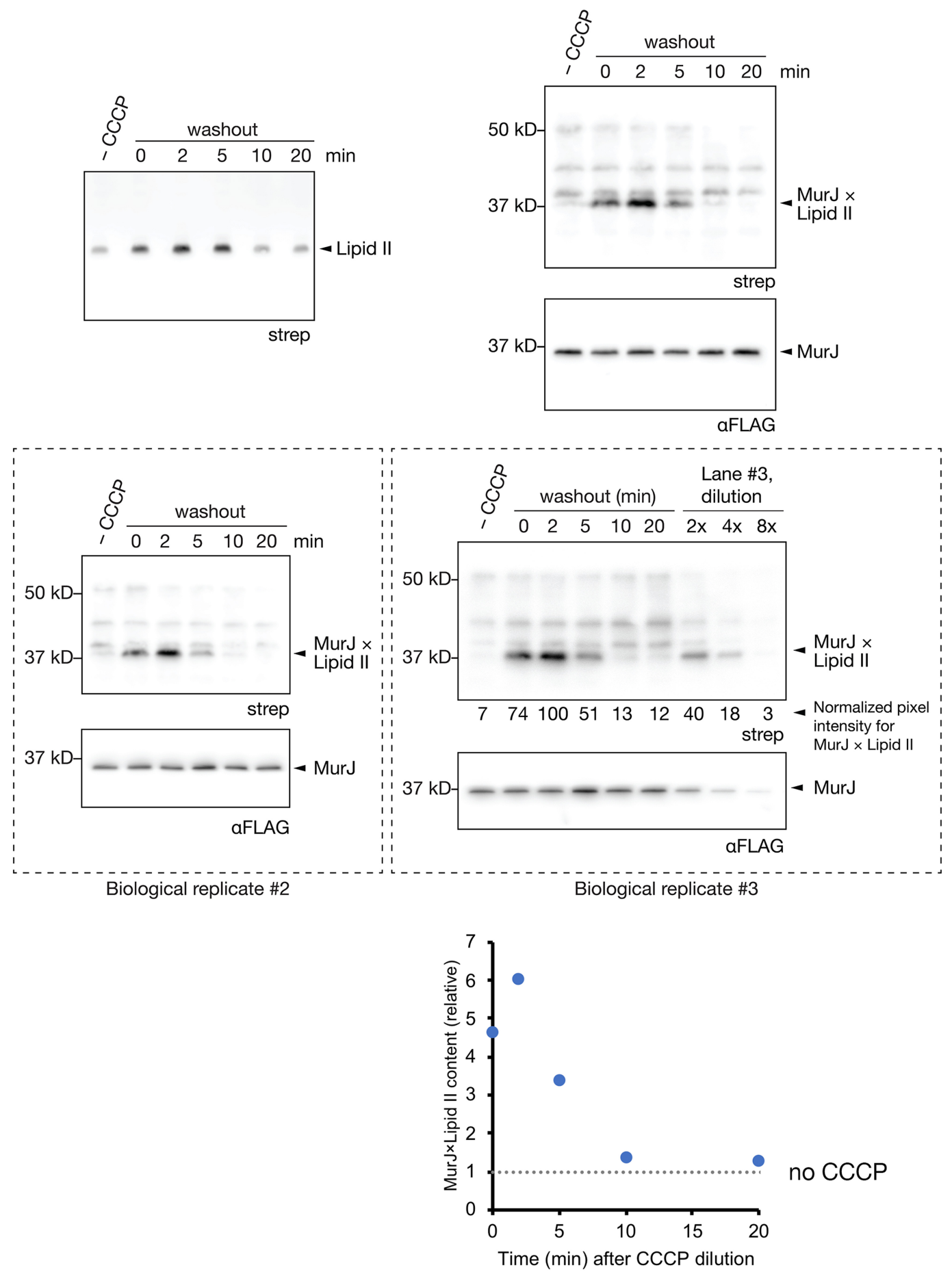


Supplementary figure S1 1. Treatment with $1 \mu \mathrm{M}$ CCCP does not accumulate MurJ $\times$ Lipid II. Cultures of cells expressing MurJ ${ }^{\mathrm{F} 22 p \mathrm{Bpa}}$ were treated with DMSO or $100 \mu \mathrm{M}$ CCCP and subsequently diluted $100 \times$ into fresh media containing no CCCP or $1 \mu \mathrm{M}$ CCCP, irradiated, and assessed for MurJxLipid II formation by on-membrane biotinylation.

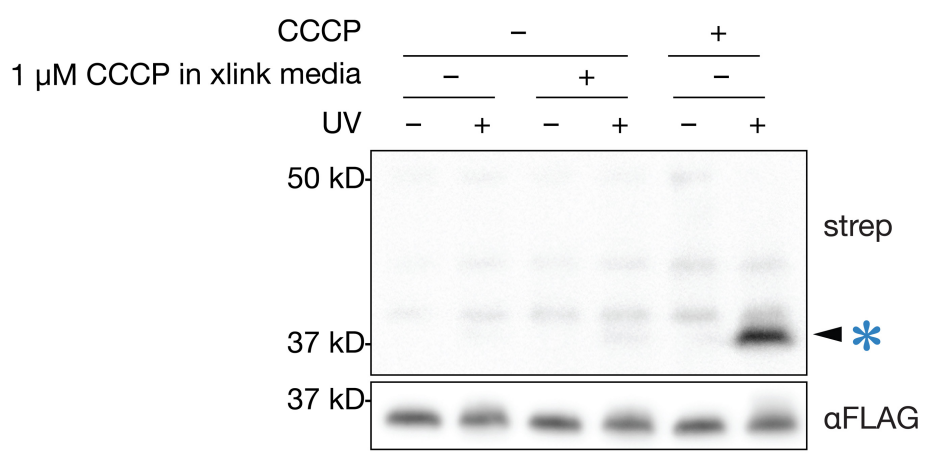


Supplementary figure S12. Uncropped western blots from Figure 3d including biological replicates and quantification of MurJ XLipid II content for the third replicate.
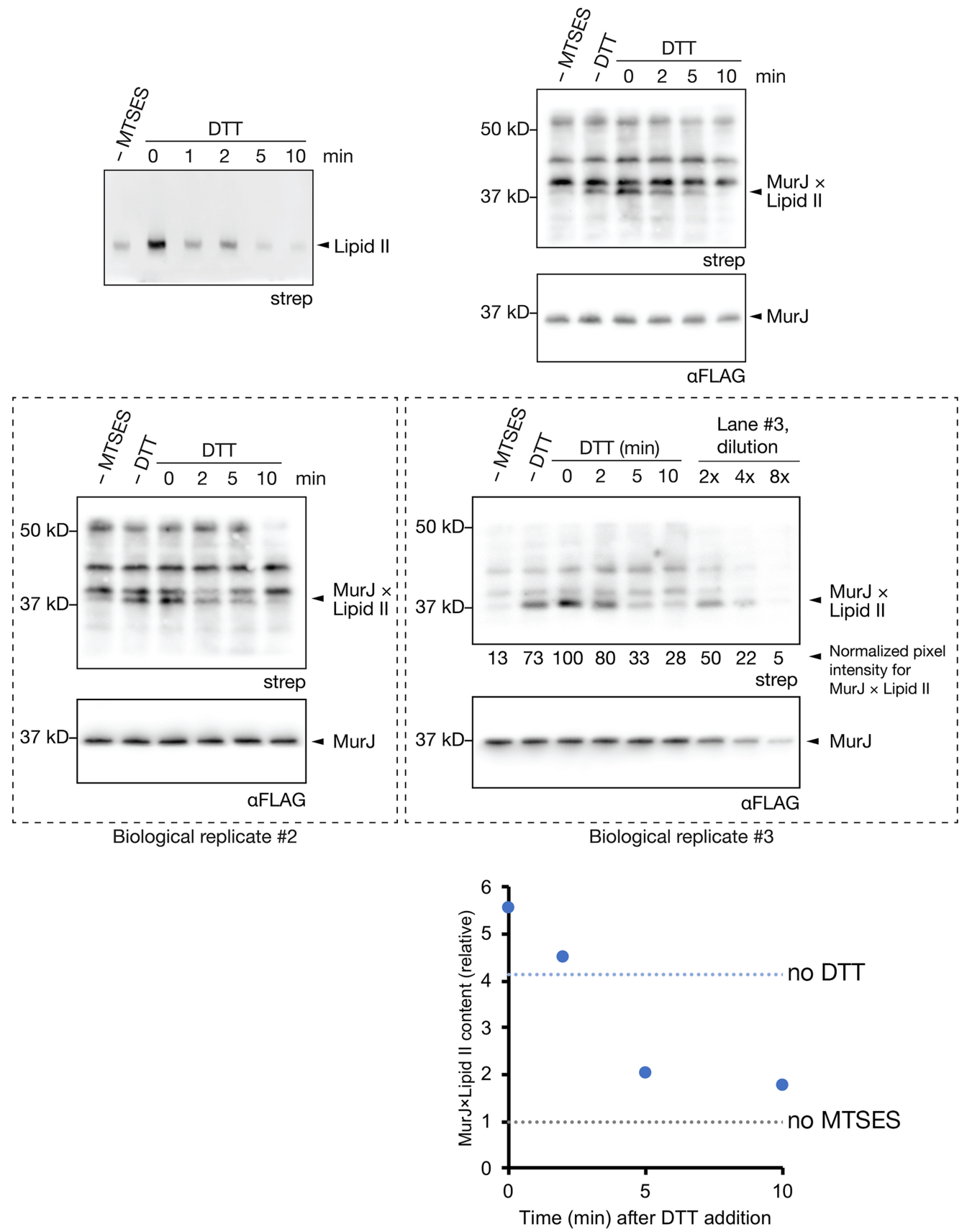
Supplementary figure S13. The diffuse membrane protein GlpT does not crosslink to Lipid II. (a) Strains expressing $p$ Bpa-containing GlpT were crosslinked alongside $\mathrm{MurJ}^{\mathrm{F} 22 \mathrm{pBpa}}$ and assessed for MurJ $\times$ Lipid II formation by on-membrane biotinylation. (b) Location of the 6 sites of $p$ Bpa incorporation in GlpT as viewed from the membrane (top) and from the periplasm (bottom).
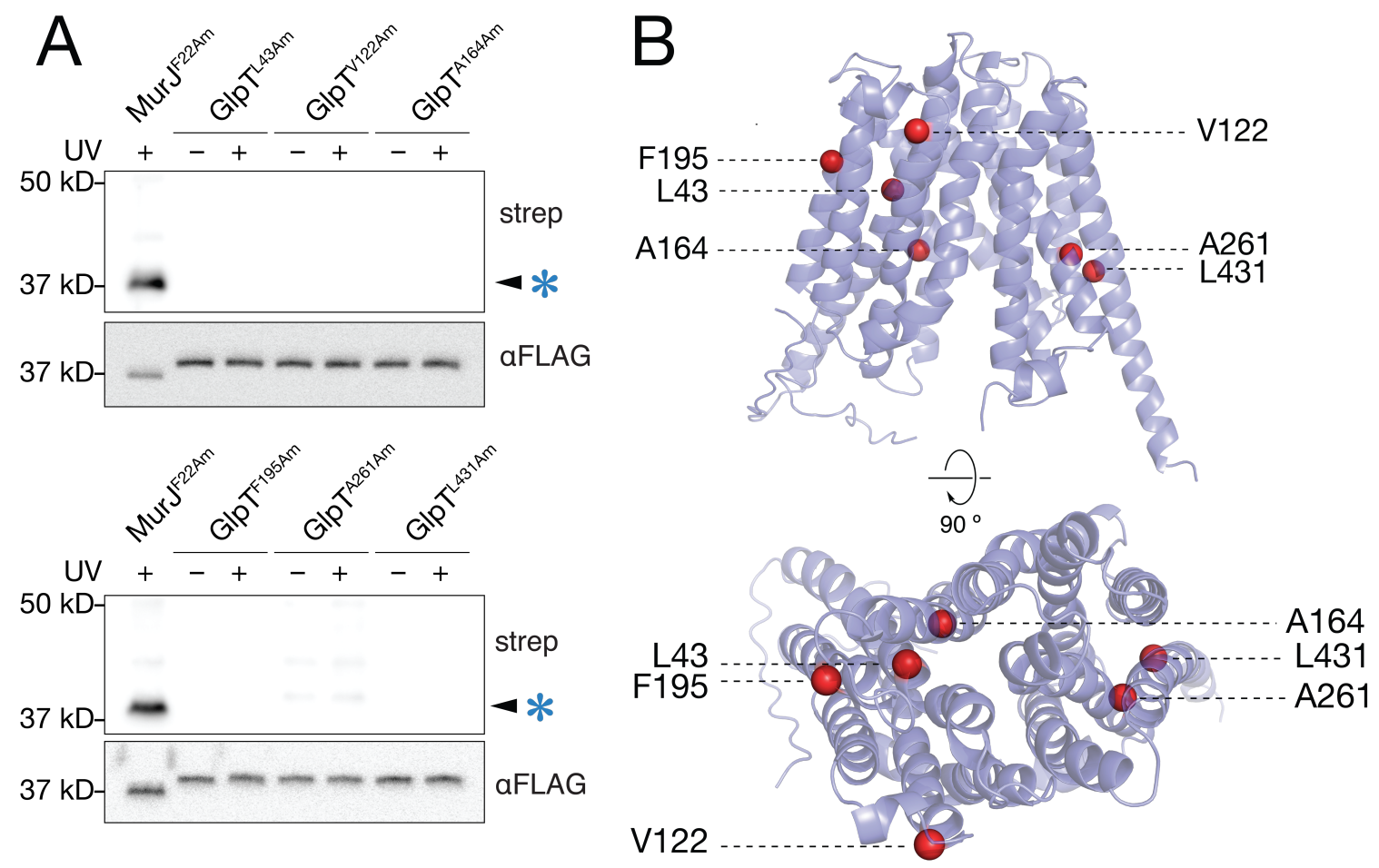
Supplementary figure S14. Uncropped blots from Figure 4c.

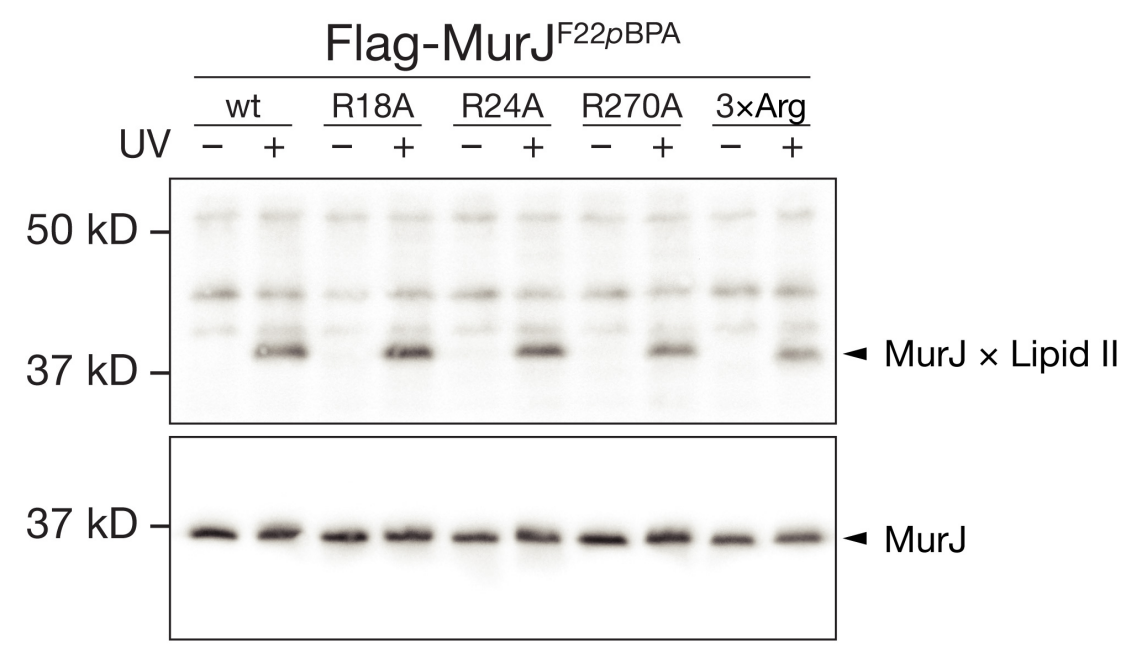


Supplementary figure S15. Uncropped blots from Figure 4d including biological replicates and quantification of MurJ×Lipid II content for the third replicate.
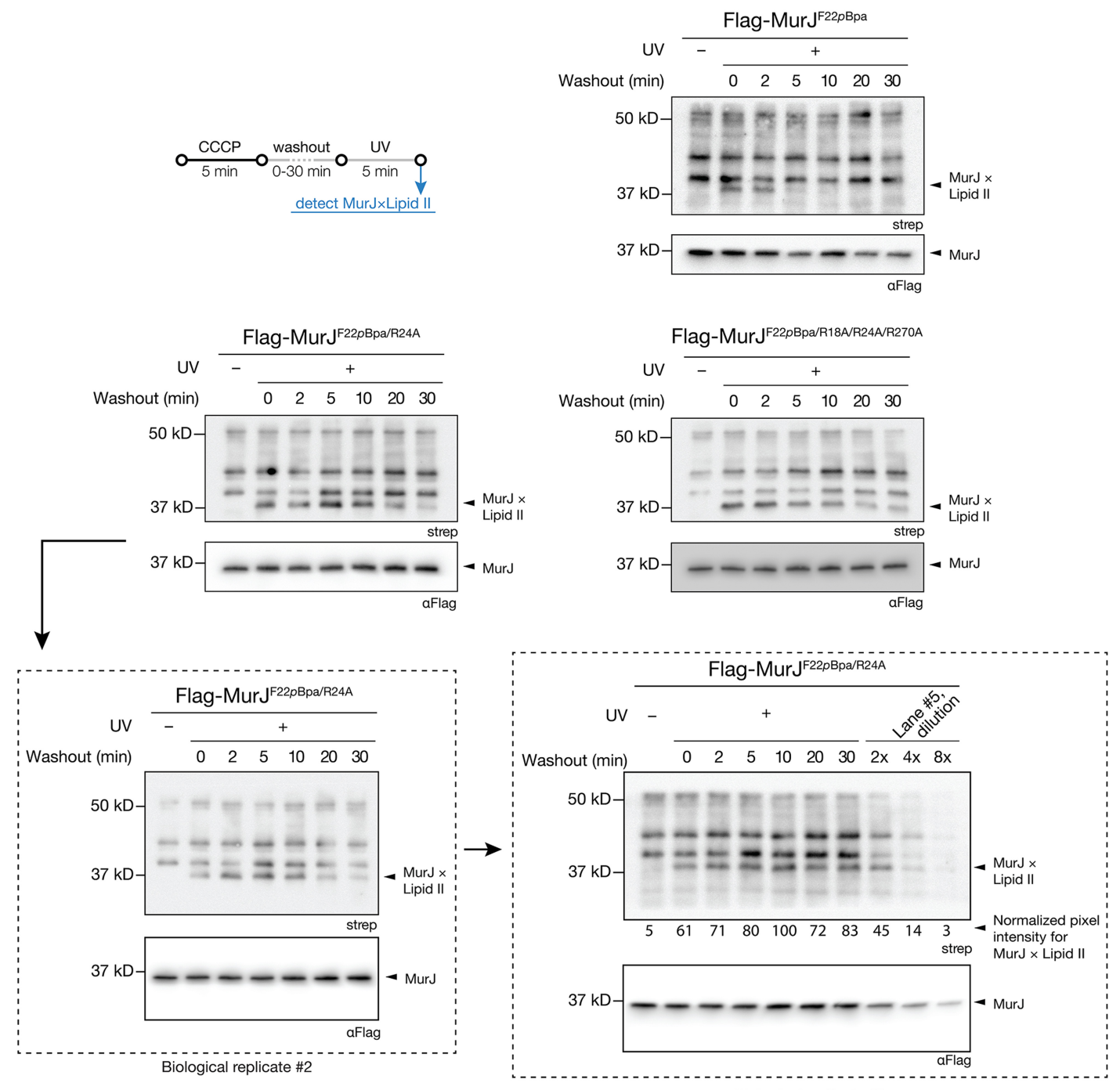

Biological replicate \#3

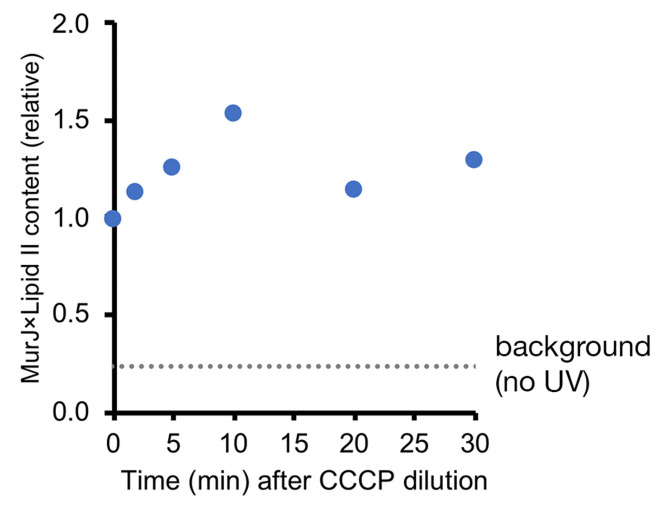


Supplementary table S1. Strains used in this study.

\begin{tabular}{clr} 
Strain & \multicolumn{1}{c}{ Genotype } & Source \\
\hline NR4128 & MG1655 $\Delta$ mur)::FRT $\Delta$ malE::(araCPARA uppS FRT) $\Delta$ lacIZYA::FRT (pRC7KanMurJ) & This study \\
NR4158 & MG1655 $\Delta$ mur)::FRT $\Delta$ malE::(araCPARA uppS FRT) $\Delta$ lacIZYA::FRT (pRC7KanMurJ; pSUP-BpaRS-6TRN) & This study \\
NR4292 & NR4158 (pET23/42FLAGMurJ $\Delta$ Cys/F22Am)* & This study \\
NR5684 & NR4158 (pET23/42FLAGMurJ $\Delta$ Cys/F22Am/A29C)* & This study \\
AM39 & NR4158 (pET23/42FLAGMurJ $\Delta$ Cys/R18A/F22Am) & This study \\
AM40 & NR4158 (pET23/42FLAGMurJ $\Delta$ Cys/F22Am/R24A) & This study \\
AM41 & NR4158 (pET23/42FLAGMurJ $\Delta$ Cys/F22Am/R270A) & This study \\
AM42 & NR4158 (pET23/42FLAGMurJ $\Delta$ Cys/R18A/F22Am/R24A/R270A) & This study \\
AM43 & NR4158 (pET23/42FLAGMurJ $\Delta$ Cys/F22Am) & This study \\
AM68 & NR4158 (pET23/42FLAGGlpT/L43Am) & This study \\
AM70 & NR4158 (pET23/42FLAGGlpT/V122Am) & This study \\
AM73 & NR4158 (pET23/42FLAGGlpT/A164Am) & This study \\
AM35 & NR4158 (pET23/42FLAGGlpT/F195Am) & This study \\
AM74 & NR4158 (pET23/42FLAGGlpT/A261Am) & This study \\
AM79 & NR4158 (pET23/42FLAGGlpT/L431Am) & This study \\
\hline Plum
\end{tabular}

* Plasmid pRC7KanMurJ was lost from this strain as described in the section Construction and growth of Amber library 
Supplementary table S2. Plasmids used in this study.

\begin{tabular}{|c|c|c|}
\hline Plasmid ID & Description & Source \\
\hline- & pET23/42FLAGMurJ $\Delta$ Cys/F22Am & This study \\
\hline - & pET23/42FLAGMurJ $\Delta$ Cys/F22Am/A29C & This study \\
\hline pAM052 & pET23/42FLAGMurJ $\Delta$ Cys/R18A/F22Am & This study \\
\hline pAM053 & pET23/42FLAGMurJ $\Delta$ Cys/F22Am/R24A & This study \\
\hline pAM054 & pET23/42FLAGMurJ $\Delta$ Cys/F22Am/R270A & This study \\
\hline pAM056 & pET23/42FLAGMurJ $\Delta$ Cys/R18A/F22Am/R24A/R270A & This study \\
\hline pAM087 & pET23/42FLAGGlpT/L43Am & This study \\
\hline pAM089 & pET23/42FLAGGlpT/V122Am & This study \\
\hline pAM092 & pET23/42FLAGGlpT/A164Am & This study \\
\hline pAM048 & pET23/42FLAGGlpT/F195Am & This study \\
\hline pAM093 & pET23/42FLAGGlpT/A261Am & This study \\
\hline pAM098 & pET23/42FLAGGlpT/L431Am & This study \\
\hline _- & pSUP-BpaRS-6TRN & Ref. 1 \\
\hline _ & pRC7KanMurJ & Ref. 2 \\
\hline
\end{tabular}




\section{Materials}

Biotin-D-lysine (BDL) and PBP4[Y21-Q383] were prepared as previously described. ${ }^{3}$ PBP5[D30-N385] was prepared as previously described ${ }^{4}$ Sodium (2-sulfonatoethyl)methanethiosulfonate (MTSES) was purchased from Toronto Research Chemicals. 4-Benzoyl-L-phenylalanine ( $p \mathrm{Bpa}$ ) was obtained from Bachem. 6Aza-2-thiothymine was purchased from Alfa Aesar. M2 ${ }^{\circledR}$-HRP was fluorescently tagged using AzureSpectra Antibody Labeling Kit 700 (AC2188). IRDye 800CW Streptavidin was purchased from LI-COR. StreptavidinHRP was purchased from KPL. LB Miller media and agar were purchased from Becton Dickinson. All other chemicals were purchased from Sigma-Aldrich. Glucose (0.2\%) M63 minimal broth and agar were prepared as described previously..$^{5}$ All liquid cultures were grown under aeration at $37^{\circ} \mathrm{C}$ unless otherwise noted. Growth was monitored by measuring the optical density at $600 \mathrm{~nm}\left(\mathrm{OD}_{600}\right)$. Western blotting was performed on a 0.2 $\mu \mathrm{M}$ Immun-Blot ${ }^{\oplus}$ PVDF membrane (Bio-Rad). HRP-conjugated antibodies were visualized using Amersham ECL Prime Western Blotting Detection Reagent. UV irradiation was carried out via a Blak-Ray ${ }^{\oplus}$ B-100AP HighIntensity UV Lamp, 365 nm (UVP, Upland, CA). Structures were visualized in PyMOL. ${ }^{6}$

\section{Construction and growth of Amber library}

The MurJ-Amber library was constructed by site-directed mutagenesis to introduce the TAG codon into the coding region of plasmid pET23/42FLAGMurJ $\Delta$ Cys (Ref. 7) as a template. Competent cells of strain NR4128 (MG1655 $\Delta$ murJ::FRT $\Delta m a l E::(a r a C$ PARA Upps FRT) $\Delta$ lacIZYA::FRT (pRC7KanMurJ)) were transformed with the pET23/42FLAGMurJ $\triangle$ Cys/Am library and plated on LB agar supplemented with carbenicillin $(50 \mu \mathrm{g} / \mathrm{mL})$ as a selection marker. The plasmid pRC7KanMurJ (Ref. 2) has a partitioning defect (which causes it to be easily lost in the presence of a functional murJ allele) and encodes a wild-type murJ allele in addition to the enzyme $\beta$-galactosidase. Colonies grown on agar supplemented with 5-bromo-4-chloro-3-indolyl $\beta$-Dgalactopyranoside (X-Gal) will therefore appear blue unless another functional allele of murJ is present. Transformants of NR4128 with pET23/42FLAGMurJ $\Delta$ Cys/Am were thus grown in liquid LB media supplemented with carbenicillin $(50 \mu \mathrm{g} / \mathrm{mL})$, made competent by resuspension in TSS buffer, ${ }^{8}$ transformed with the plasmid pSup-BpaRS-6TRN (Ref. 1), which encodes the tRNA and corresponding aminoacyl-tRNA synthetase for $p B$ pa, and plated on LB supplemented with carbenicillin $(50 \mu \mathrm{g} / \mathrm{mL})$ and chloramphenicol $(30 \mu \mathrm{g} / \mathrm{mL})$ to retain the pET23/42FLAGMurJ $\Delta$ Cys and pSup-BpaRS-6TRN plasmids, respectively, and X-gal ( $150 \mu \mathrm{g} / \mathrm{mL})$. White colonies, indicating complementation by the MurJ ${ }^{\mathrm{Am}}$ variant, were purified on LB agar supplemented with carbenicillin $(50 \mu \mathrm{g} / \mathrm{mL})$, chloramphenicol $(30 \mu \mathrm{g} / \mathrm{mL}), p B p a(480 \mu \mathrm{M})$, and X-gal $(150 \mu \mathrm{g} / \mathrm{mL})$. pBpadependent expression was confirmed by assessing Flag-MurJ levels in cultures grown in the presence or absence of $p \mathrm{Bpa}(480 \mu \mathrm{M})$ via western blot $\left(\mathrm{M}^{\circledR} \alpha\right.$ Flag $)$. In the case of $\mathrm{R} \rightarrow \mathrm{A}$ mutant $\mathrm{pET} 23 / 42$ vectors, which encode nonfunctional MurJ, a similar strain construction protocol to the above was carried out, except that no pRC7KanMurJ loss is possible, requiring a merodiploid strain. As such, X-gal was omitted, and transformation of pET23/42FLAGMurJ $\Delta$ Cys $(R \rightarrow A)$ into NR4128 was followed by transformation with pSup-BpaRS-6TRN. The same selection markers were used as described above, taking care to add kanamycin for AM43 to retain 
pRC7KanMurJ. For GlpT, the homogeneously diffuse inner-membrane glycerol-3-phosphate transporter, ${ }^{9-10}$ a pET23/42FLAGGlpT vector and merodiploid Amber library was similarly constructed by transformation into NR4128.

\section{Extraction and detection of Lipid II from cultures}

Lipid II extraction protocol was adapted from previously published protocols., ${ }^{31}$ Aliquots $(1 \mathrm{~mL})$ of cultures were pipetted into $3.5 \mathrm{~mL}$ of a mixture of methanol and chloroform (2:1) in borosilicate glass culture tubes (16 $\times 100 \mathrm{~mm}$ ) and shaken for $2 \mathrm{~min}$ at $1000 \mathrm{rpm}, 25^{\circ} \mathrm{C}$ in a ThermoMixer $\mathrm{C}^{\odot}$ (Eppendorf) to form a single phase. Cell debris was pelleted by centrifugation for $2 \mathrm{~min}$ at $2000 \times \mathrm{g}$. To the supernatant, $2 \mathrm{~mL}$ chloroform was added, followed by acidification with $0.1 \mathrm{~N} \mathrm{HCl}$ to $\mathrm{pH} 1$, as determined by $\mathrm{pH}$ indicator strips. Samples were shaken and centrifuged as above to form a two-phase system. As much of the aqueous upper layer was removed without disturbing the interface between the aqueous and organic phases, and $1 \mathrm{~mL}$ methanol was subsequently added to form a single liquid phase upon shaking. Samples were transferred to $1.5-\mathrm{mL}$ conical microcentrifuge tubes by glass pipette, then dried by nitrogen stream at $40{ }^{\circ} \mathrm{C}$. Dried samples were dissolved in $150 \mu \mathrm{L}$ of a mixture of methanol and chloroform $(2: 1)$ by shaking for 2 minutes at $2000 \mathrm{rpm}, 25^{\circ} \mathrm{C}$, then centrifuged at 21,000 $\times g$ for 1 minute and dried by nitrogen stream at $40^{\circ} \mathrm{C}$. This was repeated with $40 \mu \mathrm{L}$ organic mixture, then crude lipid extracts were dissolved in $10 \mu \mathrm{L} \mathrm{DMSO}$ by shaking $10 \mathrm{~min}$ at 500,1000 , and $2000 \mathrm{rpm}, 50^{\circ} \mathrm{C}$. Extracts were stored at $-20^{\circ} \mathrm{C}$.

Crude Lipid II extracts $(2 \mu \mathrm{L})$ were added to $12.5 \mathrm{mM}$ HEPES-NaOH (pH 7.5), $100 \mu \mathrm{M} \mathrm{MnCl}, 250 \mu \mathrm{M}$ Tween 80, $3 \mathrm{mM}$ BDL, $8 \mu \mathrm{M}$ PBP4[Y21-Q383] (10 $\mu \mathrm{L}$ total volume), then incubated $1 \mathrm{~h}$ at $25^{\circ} \mathrm{C}$ to biotinylate Lipid II. Reactions were quenched by the addition of $10 \mu \mathrm{L}$ of $2 \times$ SDS sample buffer and shaking. SDS samples $(3 \mu \mathrm{L})$ were loaded onto a $14 \%$ polyacrylamide gel ( $\mathrm{pH} 8.8$ ) and run for $40 \mathrm{~min}$ at a constant $30 \mathrm{~mA}$ current using a running buffer composed of $25 \mathrm{mM}$ Tris, $192 \mathrm{mM}$ glycine, $0.1 \%$ SDS. Samples were transferred onto PVDF membranes at $10 \mathrm{~V}$ for $1 \mathrm{~h}$, using a transfer buffer composed of $25 \mathrm{mM}$ Tris, $192 \mathrm{mM}$ glycine. Membranes were blocked $20 \mathrm{~min}$ in StartingBlock ${ }^{\mathrm{TM}}$ Buffer (Thermo \#375378) then incubated $1 \mathrm{hr}$ in a 1:10,000 dilution of streptavidin-HRP in TBS $+0.02 \%(\mathrm{w} / \mathrm{v})$ Tween 20 followed by washing in the same buffer $(8 \times$ $10 \mathrm{~min}$ ). Washed membranes were treated with ECL Reagent and visualized using an Azure c400 imaging system (Azure Biosystems, Inc., Dublin, CA).

\section{Capture of MurJ×Lipid II by photocrosslinking}

$30 \mathrm{~mL}$ cultures of Amber library strains were grown to mid-log phase $\left(\mathrm{OD}_{600} \approx 0.3\right)$ in $\mathrm{M} 63$ minimal medium supplemented with $30 \mu \mathrm{g} / \mathrm{mL}$ chloramphenicol, $50 \mu \mathrm{g} / \mathrm{mL}$ carbenicillin $(+50 \mu \mathrm{g} / \mathrm{mL}$ kanamycin for AM3943), $480 \mu \mathrm{M} \mathrm{pBpa}$, and then pelleted $(4,000 \times g, 6 \mathrm{~min})$. The pellet was resuspended in $900 \mu \mathrm{L}$ of the same media at $37^{\circ} \mathrm{C}$ and incubated for $5 \mathrm{~min}$ at $37^{\circ} \mathrm{C}$ with shaking ( $550 \mathrm{rpm}$, ThermoMixer $\left.\mathrm{C}^{\circledast}\right)$. CCCP was added to $100 \mu \mathrm{M}$ final (from a $10 \mathrm{mM}$ stock in DMSO) and the cultures were incubated for another $5 \mathrm{~min}$ at $37^{\circ} \mathrm{C}$ with 
shaking and then shifted to $16^{\circ} \mathrm{C}$ for an additional $10 \mathrm{~min} .450 \mu \mathrm{L}$ aliquots of CCCP-treated cultures were diluted into $45 \mathrm{~mL}$ of the same media (pre-cooled to $16^{\circ} \mathrm{C}$ ) and irradiated with UV light for 5 min at room temperature with gentle stirring. Prior to UV irradiation, a $1 \mathrm{~mL}$ aliquot of cells was taken for Lipid II level quantification (see Extraction and detection of Lipid II from cultures). Non-photocrosslinked controls were covered with aluminum foil during this step. Irradiated samples and controls were then collected $\left(8,000 \times g, 6 \mathrm{~min}, 4^{\circ} \mathrm{C}\right)$ and pellets frozen at $-80^{\circ} \mathrm{C}$. For inhibition with MTSES, the procedure was performed as described, with the exception that OD 1 cultures were treated with $400 \mu \mathrm{M}$ MTSES for $5 \mathrm{~min}$ at $37^{\circ} \mathrm{C}$ before dilution into media supplemented with $10 \mathrm{mM}$ DTT. For pre-treatment with fosfomycin, OD 0.3 cultures were resuspended to OD 1 in M63 media supplemented with $0.23 \mathrm{M}$ sucrose (osmoprotectant). Following 45 min treatment with 100 $\mu \mathrm{g} / \mathrm{mL}$ fosfomycin, cells were treated with CCCP and cross-linked as above.

\section{On-membrane labeling and detection of MurJ $\times$ Lipid II}

Cell pellets were thawed on ice, resuspended in $9 \mathrm{~mL}$ of $4{ }^{\circ} \mathrm{C}$ lysis buffer $(50 \mathrm{mM} \mathrm{HEPES} \mathrm{pH} \mathrm{7.5,} 150 \mathrm{mM}$ $\mathrm{NaCl}, 20 \mathrm{mM} \mathrm{MgCl} 2,0.5 \mathrm{mM}$ PMSF, $5 \mathrm{mM} \mathrm{DTT}$ ), and lysed by sonication ( $2 \mathrm{~min}$ in 10 -sec pulses). The lysates were pelleted for $10 \mathrm{~min}$ at $8,000 \times \mathrm{g}, 4^{\circ} \mathrm{C}$ and the supernatants further pelleted for $1.5 \mathrm{~h}$ at $100,000 \times \mathrm{g}$. The resulting pellets were resuspended in $100 \mu \mathrm{L}$ of $1 \times$ SDS buffer. For $\alpha$ Flag blots, $2 \mu \mathrm{L}$ of resuspended membranes were run on a 4-14\% gradient gel for $45 \mathrm{~min}$ at $200 \mathrm{~V}$, and transferred to PVDF membrane (25 V, 20 min). The membrane was blocked for $20 \mathrm{~min}$ (StartingBlock ${ }^{\mathrm{TM}}$ PBS buffer, ThermoFisher), washed $1 \times 1 \mathrm{~min}$ with TBST (20 mM Tris, $150 \mathrm{mM} \mathrm{NaCl}, 0.1 \%$ Tween 20, $\mathrm{pH} 7.5$ ), and incubated with antibody for $1 \mathrm{~h}$ (10,000-fold dilution of Monoclonal ANTI-FLAG M2 ${ }^{\circledR}$-HRP, Sigma). Following $8 \times 10$ min washes with TBST, the membrane was visualized by chemiluminescence. For on-membrane BDL exchange reactions, $10 \mu \mathrm{L}$ of resuspended membranes were run on a $10 \%$ gel ( $4 \%$ stacking layer) for $2.5 \mathrm{~h}$ at $120 \mathrm{~V}$, and transferred to PVDF membrane (25 V, $20 \mathrm{~min}$ ). The membrane was blocked for $20 \mathrm{~min}$ (StartingBlock ${ }^{\mathrm{TM}}$ PBS buffer, Thermo Fisher), washed $1 \times 1$ min with TBST, and incubated in BDL exchange mixture ( $3 \mathrm{mM} \mathrm{BDL}, 8 \mu \mathrm{M}$ S. aureus PBP4[Y21-Q383], in 12.5 mM HEPES pH 7.5, $100 \mu \mathrm{M} \mathrm{MnCl}_{2}, 250 \mu \mathrm{M}$ Tween 80). The membrane was then washed with TBST for $10 \times 10 \mathrm{~min}$ and incubated for $1 \mathrm{~h}$ with streptavidin-HRP (10,000-fold dilution of streptavidin-HRP from KPL). Following $8 \times 10$ min washes, the membrane was visualized by chemiluminescence. Western blot quantification was performed using Image J. ${ }^{12} \mathrm{~A}$ twofold dilution series of the strongest intensity band was prepared and run alongside samples to be quantified. A standard curve was prepared from this dilution series, fit to a linear trendline $\left(\mathrm{R}^{2}\right.$ values of $0.9965,0.9972$, and 0.9989 for Figures S10, S12, and S15, respectively), and used to convert band intensity to relative MurJ×Lipid II amount for each sample. For PBP5 treatment, membranes were transferred and blocked as described above, then incubated with a $10 \mu \mathrm{M}$ solution of PBP5[D30-N385] in TBST for 30-75 min. The membrane was then washed with TBST for $2 \times 1$ min and treated with the BDL/PBP4 mixture as detailed previously. 


\section{Purification of Flag-MurJ}

Cell pellets were thawed on ice, resuspended in $9 \mathrm{~mL}$ of $4{ }^{\circ} \mathrm{C}$ lysis buffer $(50 \mathrm{mM} \mathrm{HEPES} \mathrm{pH} \mathrm{7.5,} 150 \mathrm{mM}$ $\mathrm{NaCl}, 20 \mathrm{mM} \mathrm{MgCl} 2,0.5 \mathrm{mM}$ PMSF, $5 \mathrm{mM} \mathrm{DTT}$ ), and lysed by sonication ( $2 \mathrm{~min}$ in 10-sec pulses). The lysates were pelleted for $10 \mathrm{~min}$ at $8,000 \times \mathrm{g}, 4^{\circ} \mathrm{C}$ and the supernatants further pelleted for $1.5 \mathrm{~h}$ at $100,000 \times \mathrm{g}$. The resulting pellets were resuspended in $50 \mu \mathrm{L}$ of $50 \mathrm{mM}$ HEPES, $250 \mathrm{mM} \mathrm{NaCl}, 1 \% \mathrm{DDM}$, and incubated for $3 \mathrm{~h}$ at $500 \mathrm{rpm}, 4^{\circ} \mathrm{C}$. Meanwhile, the $\mathrm{M}^{\circledR}{ }^{\circledR}$ column $(250 \mu \mathrm{L}$ of settled resin), stored in $1 \times$ HBS (20 mM HEPES $\mathrm{pH} 7.5,250 \mathrm{mM} \mathrm{NaCl})+0.02 \% \mathrm{NaN}_{3}$, was cleaned with $500 \mu \mathrm{L}$ of $100 \mathrm{mM}$ sodium citrate $\mathrm{pH}$ 3. The low $\mathrm{pH}$ was then quenched via addition of $1 \mathrm{~mL}$ of $1 \times \mathrm{HBS}$, and the column equilibrated with $1 \mathrm{~mL}$ wash buffer $(20$ mM HEPES, $500 \mathrm{mM} \mathrm{NaCl}, 0.1 \%$ DDM). $450 \mu \mathrm{L}$ of wash buffer were added to the sample, which was then added to the column. The flow-through was re-applied twice. After $2 \times 500 \mu \mathrm{L}$ washes with wash buffer, the Flag-labeled MurJ was eluted with $5 \times 100 \mu \mathrm{L}$ of elution buffer $(20 \mathrm{mM}$ HEPES, $500 \mathrm{mM} \mathrm{NaCl}, 0.1 \%$ DDM, 0.2 $\mathrm{mg} / \mathrm{mL}$ Flag peptide). The eluate was concentrated as appropriate using a $50 \mathrm{kDa}$ MWCO spin filter $(5,000 \times$ $g$ ) and added to SDS-PAGE loading buffer in preparation for Western blotting.

\section{SUPPORTING INFORMATION REFERENCES}

(1) Ryu, Y.; Schultz, P. G. Efficient incorporation of unnatural amino acids into proteins in Escherichia coli. Nat Methods. 2006, 3, 263-265.

(2) Butler, E. K.; Davis, R. M.; Bari, V.; Nicholson, P. A.; Ruiz, N. Structure-function analysis of MurJ reveals a solvent-exposed cavity containing residues essential for peptidoglycan biogenesis in Escherichia coli. J. Bacteriol. 2013, 195, 4639-4649.

(3) Qiao, Y.; Lebar, M. D.; Schirner, K.; Schaefer, K.; Tsukamoto, H.; Kahne, D.; Walker, S. Detection of lipidlinked peptidoglycan precursors by exploiting an unexpected transpeptidase reaction. J. Am. Chem. Soc. 2014, $136,14678-14681$.

(4) Lupoli, T. J.; Tsukamoto, H.; Doud, E. H.; Wang, T. S.; Walker, S.; Kahne, D. Transpeptidase-mediated incorporation of D-amino acids into bacterial peptidoglycan. J. Am. Chem. Soc. 201 1, 133, 10748-10751.

(5) Silhavy, T. J.; Berman, M. L.; Enquist, L. L. W. W. Experiments with gene fusions. Cold Spring Harbor Laboratory. 1984.

(6) The PyMOL Molecular Graphics System, Version 2.0 Schrödinger, LLC.

(7) Kumar, S.; Rubino, F. A.; Mendoza, A. G.; Ruiz, N. The bacterial lipid II flippase MurJ functions by an alternating-access mechanism. J. Biol. Chem. 2019, 294, 981-990.

(8) Chung, C. T.; Niemela, S. L.; Miller, R. H. One-step preparation of competent Escherichia coli: transformation and storage of bacterial cells in the same solution. Proceedings of the National Academy of Sciences. 1989, 86, 2172-2175.

(9) Oswald, F.; Varadarajan, A.; Lill, H.; Peterman, E. J.; Bollen, Y.J. MreB-Dependent Organization of the E. coli Cytoplasmic Membrane Controls Membrane Protein Diffusion. Biophys. J. 2016, 110, 1139-1149.

(10) Liu, X.; Meiresonne, N. Y.; Bouhss, A.; den Blaauwen, T. FtsW activity and lipid II synthesis are required for recruitment of MurJ to midcell during cell division in Escherichia coli. Mol. Microbiol. 2018, 109, 855-884.

(11) Guan, Z.; Breazeale, S. D.; Raetz, C. R. Extraction and identification by mass spectrometry of undecaprenyl diphosphate-MurNAc-pentapeptide-GlcNAc from Escherichia coli. Anal. Biochem. 2005, 345, 336-339. 
(12) Schneider, C. A.; Rasband, W. S.; Eliceiri, K. W. NIH Image to ImageJ: 25 years of image analysis. Nat Methods. 2012, 9, 671-675. 